\title{
INDICADORES NA ESTIMATIVA DO FLUXO DE DIGESTA EM BOVINOS DE CORTE\#
}

\author{
MARKERS FOR ESTIMATE BEEF CATTLE DIGESTA FLOW
}

\author{
Queiroz, M.F.S. ${ }^{1 \mathrm{~A}}$, Morais, J.A.S. ${ }^{1 \mathrm{~B}}$, Berchielli, T.T. ${ }^{1 \mathrm{C}}$, Malheiros, E.B. ${ }^{1 \mathrm{D}}$, Sader, A.P.O. ${ }^{1 \mathrm{E}}$, \\ Resende, de K.T. ${ }^{1 F}$ e Eifert, E.C. ${ }^{2}$
}

\begin{abstract}
${ }^{1}$ Faculdade de Ciências Agrárias e Veterinárias. UNPESP. Jaboticabal. São Paulo. Brasil. Amfernanda_queiroz@yahoo.com.br; Bjuci_morais@yahoo.com.br; cttberchi@fcav.unesp.br; Deuclides@fcav.unesp.br; Ebrasil.anasader@fcav.unesp.br; Fkresende@fcav.unesp.br EMBRAPA Arroz e Feijão. Santo Antonio de Goiás. Goiás. Brasil. eifert@cnpaf.embrapa.br
\end{abstract}

\section{PalaVRas ChaVe adicionais}

Alcanos. CoEDTA. Cromio. Digesta duodenal. FDNi. FDAi. Omasal.

\section{RESUMO}

O objetivou-se comparar os métodos de único e duplo indicador na estimativa do fluxo de digesta duodenal e omasal em 7 novilhos Nelore (peso medio $250 \mathrm{~kg}$ ), canulados no rúmen e duodeno. Os animais receberam via infusão ruminal contínua por bomba solução contendo CoEDTA. Também receberam uma cápsula contendo $\mathrm{Cr}_{2} \mathrm{O}_{3}$ através da cânula ruminal, e uma cápsula contendo alcanos externos $\mathrm{C}_{32}, \mathrm{C}_{36}$. Indicadores internos fibra em detergente neutro e fibra em detergente ácido (FDNi e FDAi após $144 \mathrm{~h}$ de incubação in situ) e alcanos internos $\mathrm{C}_{31}, \mathrm{C}_{33}, \mathrm{C}_{35}$ eram parte integrante da dieta. Os novilhos foram alimentados com Brachiaria brizantha cv. marandu aos 30 ou 60 dias de rebrota. O desenho experimental para o fluxo duodenal foi em esquema fatorial $2 \times 2 \times 12 \mathrm{e}$ na estimativa do fluxo de digesta omasal o esquema fatorial foi $2 \times 2 \times 4$. Na estimativa do fluxo duodenal de MS os indicadores FDNi, CoEDTA e a combinação Co+FDNi foram eficientes na estimativa do fluxo omasal de MS se mostraram eficientes os indicadores FDNi, FDAi e a combinação Co+FDAi. O método de coleta de duplo indicador é o mais adequado para estimativa de fluxo considerando-se a digestibilidade ruminal da fibra.

\section{SUMMARY}

The duodenal and omasal dry matter flows by

\#Pesquisa financiada pela Fapesp.

\section{AdDitiOnAL KEYWORDS}

Chromium. CoEDTA. Duodenal flow. iADF. iNDF. $\mathrm{N}$-alkanes. Omasal flow.

single and double marker methods were compared in seven Nellore steers with average body weight $250 \mathrm{~kg}$, and previously canullated in the rumen and duodenum. The animals were fitted with continuous-infusion pump that administered CoEDTA solutions intraruminally. In addition, a capsule containing $\mathrm{Cr}_{2} \mathrm{O}_{3}$ and other, containing external n-alkanes $\mathrm{C}_{32}, \mathrm{C}_{36}$ were inserted into the rumen of each steer. Internal markers indigestible neutral-detergent fiber and acid-detergent fiber (iNDF and iADF after $144 \mathrm{~h}$ in situ incubation) and internal n-alkanes $\mathrm{C}_{31}, \mathrm{C}_{33}, \mathrm{C}_{35}$ were components of the diet. Steers were fed with palisade grass (Brachiaria brizantha cv. marandu) in two age of regrowth, 30 and 60 days. The duodenal flow experimental design was a factorial $2 \times 2 \times 12$ while omasal experimental design was a factorial $2 \times 2 \times 4$. Markers iNDF, CoEDTA and the combination Co+iNDF were efficacious to estimate duodenal dry matter flow whereas iNDF, iADF and the Co+iADF combination were all efficacious to predict omasal dry matter flow. In conclusion, the double marker method for estimation of omasal and duodenal dry matter flow was the most appropriate considering the ruminal fiber digestibility.

\section{INTRODUÇÃO}

Com o intuito de aumentar a eficiência de 
utilização dos nutrientes em ruminantes tem sido desenvolvidos sistemas de energia e proteína metabolizável baseados em dados derivados de estudos que medem o destino dos nutrientes no rúmen e a disponibilização de nutrientes para o animal e, a confiabilidade destes sistemas em predizer o suprimento de nutrientes para o desenvolvimento do animal é dependente da capacidade de medir apuradamente o fluxo de nutrientes pelo trato digestivo. Portanto, aumentar a precisão das medições de fluxo é fundamental para reduzir erros na predição (Ahvenjärvi et al., 2003). Apesar dessa importância, ainda existe receio na adoção de algumas metodologias para estimativa do fluxo de nutrientes pelo trato gastrintestinal de ruminantes por as divergências na literatura.

Um dos principais problemas na determinação do fluxo de nutrientes em algum ponto do trato digestivo é a amostragem da digesta. A digesta é dividida em duas fases, líquida e sólida (Faichney, 1975), por isso uma amostragem não representativa da digesta pode acontecer quando se usa um único indicador, devido à tendência da digesta em se separar durante a coleta. $\mathrm{O}$ emprego simultâneo de indicadores que apresentam afinidade pelas diferentes fases da digesta (líquida e sólida) e o cálculo de coeficientes de reconstituição pode compensar a amostragem não representativa (Faichney, 1975).

Há alguns anos a preocupação com o bem estar animal tem aumentado, buscando-se, técnicas menos invasivas de coletas nos animais. Para a coleta de fluxo de nutrientes no trato digestivo destes, as técnicas mais utilizadas são as de cânulas T no duodeno e cânulas reentrantes omasoabomasal. Estas, porém, exigem a canulação pós-rúmen dos animais. Punia et al. (1988) obtiveram amostras de digesta ruminal através do orifício retículo omasal, via fístula ruminal, succionando a digesta com o uso de uma bomba a vácuo, posteriormente outras formas de coleta de digesta omasal foram testadas (Huhtanen et al., 1997; se- gundo Ahvenjärvi et al., 2000) e Leão et al. (2005), a técnica de coleta omasal via fístula ruminal, apresenta maiores vantagens em relação à realizada no abomaso ou no duodeno, sobretudo por ser menos invasiva.

Objetivou-se avaliar indicadores externos e internos na estimativa de fluxo de matéria seca pelo método de único e duplo indicador e comparar a coleta omasal com a coleta duodenal na estimativa do fluxo de matéria seca pelo trato gastrointestinal de bovinos alimentados a base de forragem.

\section{MATERIALE MÉTODOS}

O experimento foi desenvolvido no Setor de Avaliação de Alimentos e Digestibilidade pertencente ao Departamento de Zootecnia da FCAV/UNESP, durante os meses de Janeiro e Fevereiro de 2006, com duração de 30 dias, dividido em dois períodos experimentais de 15 dias cada.

Foram utilizados 7 bovinos Nelore castrados, com peso vivo médio de $250 \mathrm{~kg}$, com implantação prévia das cânulas no rúmen e duodeno. Durante o período de adaptação ao alimento os animais foram alojados em baias individuais de aproximadamente $12 \mathrm{~m}^{2}$ com cocho, bebedouro e piso de concreto, e durante o período de coleta experimental esses animais permaneceram em gaiolas para estudos de metabolismo com piso ripado, comedouro e bebedouros individuais e bandejas adaptadas para coleta de fezes. Inicialmente seria utilizado 8 bovinos, porém, 1 animal foi desconsiderado devido a apresentar problemas.

Os animais foram alimentados com Brachiaria brizantha cv. marandu com 30 ou 60 dias de rebrota, numa oferta de $2,5 \%$ do peso corporal do animal. A forrageira foi cortada pela manhã e fornecida aos animais no cocho as 7:00 e às 19:00 h. Durante o período em que os animais permaneciam nas gaiolas de metabolismo a alimentação era dividida ao longo do período diurno, no intervalo das 7:00 às 19:00 h, devido ao fato do cocho das gaiolas não comportar todo capim em uma única vez. O consumo médio 


\section{ESTIMATIVA DO FLUXO DE DIGESTA EM BOVINOS DE CORTE}

observado neste experimento para as idades de rebrota de 30 e 60 dias foram 4,7 e $3,8 \mathrm{~kg}$ $\mathrm{MS} /$ dia, respectivamente, com médias de consumo de matéria seca em porcentagem de peso corporal de 1,7 e $1,4 \%$. Para a forrageira aos 30 dias de rebrota foi observado consumo de $66,9 \mathrm{~g} \mathrm{MS} / \mathrm{dia}$ em peso vivo metabólico e de 57,2 g MS/dia para a forrageira aos 60 dias de rebrota.

Foram preparados 20 piquetes de Brachiaria brizantha $\mathrm{cv}$. marandu para a alimentação dos animais dentre os quais, 10 piquetes foram destinados à idade de rebrota de 60 dias e 10 piquetes destinados à idade de rebrota de 30 dias. Cada piquete possuía dimensão aproximada de 20x20 m e forneceu o volumoso necessário para alimentação dos animais por três dias. No dia do corte individual de cada piquete foi realizada adubação com urea a lanço com $80 \mathrm{~kg}$ de N/ hectare. O corte da forrageira para fornecimento aos animais foi a $20 \mathrm{~cm}$ do solo, para simular o pastejo por bovinos.

O fluxo de digesta duodenal foi analisado em esquema fatorial $2 \times 2 \times 12$ (duas idades de rebrota da forrageira $\times 2$ períodos $\times 12$ indicadores) tendo como indicadores o $\mathrm{Cr}_{2} \mathrm{O}_{3}$, o CoEDTA, o acetato de itérbio, a FDNi, a FDAi, os alcanos $\mathrm{C}_{31}, \mathrm{C}_{32}, \mathrm{C}_{33}, \mathrm{C}_{35}, \mathrm{C}_{36}$ e as combinações $\mathrm{Co}+\mathrm{Cr}$ e $\mathrm{Co}+\mathrm{FDNi}$. $\mathrm{Na}$ estimativa do fluxo de digesta omasal o esquema fatorial foi $2 \times 2 \times 4$ (duas idades de rebrota da forrageira x 2 períodos $\times 4$ indicadores) tendo como indicadores o $\mathrm{Cr}_{2} \mathrm{O}_{3}$ a FDNi, a FDAi e a combinação Co+FDAi. Devido à necessidade de se analisar os dados de 7 animais, durante o primeiro período experimental 4 animais receberam a dieta com 30 dias e 3 animais a dieta com 60 dias de rebrota e, durante o segundo período experimental, 3 animais receberam a dieta com 30 dias e 4 animais a dieta com 60 dias de rebrota.

Os primeiros 10 dias de cada período experimental foram destinados à adaptação dos animais à dieta e aos indicadores. Do $11^{\circ}$ ao $15^{\circ}$ dia foram realizadas coletas para determinação do consumo voluntário e fluxo de nutrientes pelo duodeno e omaso. No $8^{\circ}$, $9^{\circ}$ e $10^{\circ}$ dia foram realizadas as coletas de fezes, da produção total do animal, em bandejas adaptadas às gaiolas metabólicas para posterior mensuração da digestibilidade total do trato digestivo.

Os animais receberam, via cânula ruminal, $10 \mathrm{~g} /$ dia de $\mathrm{Cr}_{2} \mathrm{O}_{3}$ divididos em duas doses diárias (as 7:00 e as 19:00 h), acondicionados em papel toalha. Concomitantemente com a administração do $\mathrm{Cr}_{2} \mathrm{O}_{3}$, foi administrado aos animais dois péletes impregnados com alcanos sintéticos. Os péletes de alcanos foram confeccionados com papel filtro picado em pedaços de aproximadamente $1 \mathrm{x} 1 \mathrm{~cm}$ e impregnados com os alcanos sintéticos $\mathrm{C}_{28}\left(\mathrm{n}\right.$-octacosano), $\mathrm{C}_{32}(\mathrm{n}$ dotriacontano) e $\mathrm{C}_{36}$ (n-hexatriacontano) (>97\% de pureza, Sigma-Aldrich Corp., St. Luis, MO, EUA) de acordo com Mayes et al. (1986). No primeiro período experimental, cada pélete continha $270,78 \pm 44 \mathrm{mg}$ do alcano $\mathrm{C}_{28} ; 290,36 \pm 47 \mathrm{mg}$ do alcano $\mathrm{C}_{32}$ e $325,41 \pm 55$ $\mathrm{mg}$ do alcano $\mathrm{C}_{36}$ e no segundo período $285,31 \pm 72 \mathrm{mg} \mathrm{de}_{28} ; 301,77 \pm 45 \mathrm{mg} \mathrm{de}_{32} \mathrm{e}$ $335,06 \pm 83 \mathrm{mg}$ de $\mathrm{C}_{36}$. Cada pélete foi feito pesando $6,5 \mathrm{~g}$ de papel filtro impregnado e envolto em papel toalha. A administração do $\mathrm{Cr}_{2} \mathrm{O}_{3}$, e dos péletes impregnados com os alcanos foi realizada durante 12 dias de cada período experimental, sendo os primeiros 7 dias destinados à estabilização dos indicadores na digesta. Os cinco dias posteriores foram realizadas as coletas de fezes e de digesta duodenal, para a determinação da recuperação dos indicadores e da estimativa do fluxo de MS, respectivamente.

O CoEDTA (300 mg/dia), preparado de acordo com Úden et al., (1980), e o acetato de $\mathrm{Yb}$ (300 mg/dia) foram dissolvidos em água destilada e infundidos no rúmen via cânula em infusão contínua através de uma bomba peristáltica(PROVITEC®), ajustada para manter o fluxo de $230 \mathrm{ml} / \mathrm{h}$.

A infusão dos indicadores CoEDTA e Acetato de $\mathrm{Yb}$ foi realizada durante 7 dias, iniciando-se 2 dias antes do período de coletas e estendendo-se até o final de cada 
período experimental. A concentração desses indicadores na solução foi ajustada para representar 4 dias de infusão na adaptação, visto que para a infusão os animais necessitavam estar contidos na gaiola para estudo de metabolismo. Dessa forma, optou-se por diminuir o período destinado à estabilização dos indicadores na digesta feita por infusão contínua, porém, respeitou-se a quantidade de indicadores necessária para que o equilíbrio desses no rúmen fosse atingido. As coletas de digesta foram realizadas em dois compartimentos diferentes do trato gastrintestinal: omaso e duodeno.

As coletas de digesta duodenal foram realizadas através de cânula duodenal tipo $\mathrm{T}$, durante três dias $\left(11^{\circ}\right.$ ao $13^{\circ}$ dia do experimento), totalizando ao final 24 horas de coleta, com amostragem realizada a cada 3 horas, no seguinte esquema: $1^{\circ}$ dia amostras coletadas as 02:00, 8:00, 14:00 e 20:00 h, $2^{\circ}$ dia amostras coletadas as 2:00, 11:00, 17:00 e 23:00 h, $3^{\circ}$ dia amostras coletadas as 5:00 h. As amostras foram destinadas à analise pelo método de único indicador foram secas em estufa de ar forçado a $55^{\circ} \mathrm{C}$ imediatamente após a coleta. Para o método de duplo indicador, após a coleta, as amostras foram congeladas e posteriormente separadas em fases, através de filtragem em tecido de algodão duplo. O material que ficou retido no tecido foi considerado como a fase particulada da digesta, enquanto o material que passou pelo tecido foi considerado a fase fluida (Ahvenjärvi et al., 2003). No final de cada período experimental foi constituída uma amostra composta por animal e época para a determinação de MS, FDN, FDA e a concentração dos indicadores $\mathrm{Yb}, \mathrm{Co}, \mathrm{Cr}$, alcanos, FDNi e FDAi.

As coletas da digesta omasal foram realizadas por sucção do conteúdo no orifício retículo-omasal com uma bomba de vácuo via cânula ruminal. As amostras de digesta foram obtidas durante três dias $\left(13^{\circ}\right.$ ao $15^{\circ}$ dia do experimento), totalizando ao final 24 horas de coleta, com amostras coletadas a cada 4 horas, no seguinte esquema: $1^{\text {er }}$ dia coletadas as $13: 00 \mathrm{e} 21: 00 \mathrm{~h} ; 2^{\circ}$ dia coletadas as 5:00 e 17:00 h e $3^{\text {er }}$ dia coletadas as 1:00 e 9:00 h. As amostras foram processadas da mesma forma que as amostras de digesta duodenal. As coletas de omaso foram realizadas em dias diferentes as coletas de duodeno.

Diariamente, foram coletadas amostras da forrageira fornecida aos animais e no final de cada período experimental constituída uma amostra composta.

As amostras de forragem, sobras, fezes e digestas foram secas em estufa com ventilação forçada de $55^{\circ} \mathrm{C}$, por 72 horas e, posteriormente, moídas em moinho de facas com peneira de $1 \mathrm{~mm}$. Nessas amostras foi determinado o teor de matéria seca (MS) de acordo com AOAC (1990), descrito por Silva e Queiroz (2002). A fibra em detergente ácido (FDA) e a fibra em detergente neutro (FDN) foram determinadas com as soluções detergentes descritas por Van Soest et al. (1991), com as amostras submetidas à digestão em solução detergente, por 40 minutos em autoclave a $111^{\circ} \mathrm{C}$ e 0,5 atm (Deschamps, 1999).

Nas amostras compostas de digesta duodenal e omasal foi determinada a concentração de $\mathrm{Cr}, \mathrm{Yb}, \mathrm{Co}$, FDNi e FDAi. As concentrações de $\mathrm{Cr}$, $\mathrm{Co}$ e $\mathrm{Yb}$ nas amostras foram determinadas após digestão nitroperclórica conforme de Vega e Poppi (1997) e as leituras realizadas em espectrofotômetro de absorção atômica Varian AA 220FS com chama de ar-acetileno. As amostras foram analisadas no Laboratório de Nutrição Animal da FCAV/UNESPJaboticabal.

Os teores de FDNi e FDAi na dieta, digesta e fezes foram obtidos após incubação ruminal in situ por $144 \mathrm{~h}$, segundo Berchielli et al. (2000). Para a incubação, foram utilizados 4 bovinos com peso corporal médio de $200 \mathrm{~kg}$. As amostras foram incubadas em duplicata em sacos de náilon.

A determinação do perfil de alcanos nas amostras de alimento, fezes e digesta 


\section{ESTIMATIVA DO FLUXO DE DIGESTA EM BOVINOS DE CORTE}

duodenal foram realizadas de acordo com Mayes et al. (1986), com modificações descritas em Oliván e Osoro (1999). Amostrouse 6 péletes em cada período experimental sendo a concentração de alcanos nesses péletes determinada conforme Valiente et al. (2003). As amostras foram analisadas no Departamento de Producción Animal y Ciencia de los Alimentos da Facultad de Veterinaria, Universidad de Zaragoza, Espanha. A quantificação dos alcanos foi realizada por cromatografia gasosa em um equipamento Agilent 6890A dotado de injetor automático on-column.

O consumo e a digestibilidade aparente do capim foram calculados pela diferença da quantidade ingerida (obtido pela diferença entre o alimento fornecido e as sobras) e a quantidade excretada quantificada através da coleta total de fezes.

Na estimativa do fluxo omasal e duodenal de matéria seca pelo método de único indicador a equação em função do indicador foi:

Indicador externo:

Fluxo duodenal de MS= gramas de indicador fornecido/concentração do indicador na MS duodenal.

Indicador interno:

Fluxo duodenal de MS= MS fecal $\mathrm{x}$ indicador interno nas fezes / \% indicador interno na MS duodenal.

Na estimativa do fluxo omasal e duodenal de matéria seca pelo método de duplo indicador a equação foi (Faichney, 1993):

Fluxo duodenal de MS= dose diária de indicador $F^{*}(1+R) /$ concentração do indicador $F S$ na $\mathrm{DT}+\left(\mathrm{R}^{*}\right.$ concentração do indicador FS nas GP)

onde:

$\mathrm{FS}=$ fase sólida;

$\mathrm{FF}=$ fase fluída;

$\mathrm{DT}=$ digesta total;

$\mathrm{GP}=$ grandes partículas;

$\mathrm{R}=$ fator de reconstituição da digesta;
$R^{*}=[$ (concentração do indicador FS na DT / dose diária de indicador FS) - (concentração do indicador FF na DT / dose diária de indicador FF)] / [(concentração do indicador FF nas GP / dose diária de indicador FF) - (concentração do indicador FS nas GP / dose diária de indicador FS)].

$\mathrm{Na}$ estimativa das digestibilidades ruminal a equação utilizada foi:

Digestibilidade ruminal $(\%)=[($ consumo $(\mathrm{kg})$-fluxo estimado no duodeno $(\mathrm{kg})]$ / consumo $(\mathrm{kg})]^{*} 100$

As análises estatísticas foram realizadas pelo procedimento GLM do programa computacional SAS (1990) e na comparação de médias foi utilizado o teste de Tukey a $5 \%$ de probabilidade.

$\mathrm{Na}$ análise estatística para digestibilidade ruminal da fibra foi utilizado um teste de hipóteses, onde: $\mathrm{H}_{0}=$ digestibilidade daFDN $=100 \% ; \mathrm{H}_{1}=$ digestibilidade $\mathrm{daFDN} \neq$ $100 \%$.

\section{RESULTADOSEDISCUSSÃO}

As concentrações (tabela I) dos indicadores externos $\mathrm{Cr}$, $\mathrm{Yb}, \mathrm{Co}$, fornecidos respectivamente como óxido de $\mathrm{Cr}$, acetato de $\mathrm{Yb}$ e Co-EDTA e dos indicadores internos fibra em detergente neutro indigestível (FDNi) e fibra em detergente ácido indigestível (FDAi). Foi constatado que nos diferentes compartimentos do trato gastrintestinal, duodeno e omaso, as concentrações de um mesmo indicador foram diferentes, sendo que no duodeno as concentrações foram sempre superiores que às concentrações observadas no omaso. A menor concentração de indicadores na digesta omasal também foi relatado por Huhtanen et al. (1997) em comparação à concentração de indicadores observada nas fezes de vacas da raça Holandês.

Não foi observado efeito estatístico do período experimental $(\mathrm{p}>0,05)$ nos resultados obtidos durante o experimento.

O fluxo de matéria seca duodenal diário 
para a idade de rebrota de 30 dias da $B$. brizantha cv. marandu foi em média $1,5 \mathrm{~kg}$ de MS, estatisticamente superior $(\mathrm{p}=0,003)$ que o fluxo da mesma forragem aos 60 dias de rebrota, em média 1,3 $\mathrm{kg}$ de MS, valores médios de todos os indicadores avaliados, contudo, não há relato, na literatura, de valores de fluxo de digesta em bovinos recebendo dieta exclusiva de volumoso fresco. No intervalo proposto com as idades de corte desse experimento, o observado foi menor fluxo de matéria seca para a forrageira mais velha, visto que, com o aumento na proporção de fibra da dieta decorrente da maturidade da forrageira, há diminuição na taxa de passagem do alimento e, conseqüentemente, do fluxo de digesta. Não foi observada interação estatística $(p=0,625)$ entre as idades de rebrota do capim e o indicador utilizado na estimativa de fluxo de nutrientes.

A digestibilidade da matéria seca total no trato digestivo observada foi de 74,6 e $73,6 \%$ para as idades de rebrota de 30 e 60 dias, respectivamente, e, apesar de não haver diferença estatística entre os valores $(\mathrm{p}=$ 0,058 ) há uma tendência à maior digestibilidade total da matéria seca para o capim aos 30 dias de rebrota.

A estimativa de fluxo de digesta duodenal e digestibilidade ruminal estimada com os indicadores são apresentadas na tabela II.

No método de único indicador, os indicadores $\mathrm{Cr}_{2} \mathrm{O}_{3}$, acetato de $\mathrm{Yb}$, e os alcanos $\mathrm{C}_{31}, \mathrm{C}_{32}, \mathrm{C}_{33}, \mathrm{C}_{35}$ e $\mathrm{C}_{36}$, superestimaram o fluxo duodenal de matéria seca (tabela I), considerando-se valores médios de digestibilidade ruminal da matéria seca em torno de $65 \%$ (Valadares Filho et al., 1990), com coeficientes de $32,3 \%, 38,6 \%$, $19,3 \%, 39,8 \%, 17,5 \%, 17,3 \%$ e $30,9 \%$, respectivamente, para os indicadores citados. O indicador FDAi, apesar de estatisticamente igual ao FDNi, Co e CoEDTA-FDNi, subestimou o fluxo duodenal de matéria seca, com coeficiente de digestibilidade ruminal de $80,2 \%$, considerado alto para dietas exclusivas de forragem. Leão et al.
Tabela I. Concentração dos indicadores nos diferentes compartimentos do trato gastrintestinal. (Markers concentration in different compartments of the gastrointestinal tract).

\begin{tabular}{lcc}
\hline Indicadores $^{1}$ & Duodeno & Omaso \\
\hline Cromo & $2,47 \mathrm{~g} / \mathrm{kgMS}$ & $1,88 \mathrm{~g} / \mathrm{kgMS}$ \\
Itérbio & $0,12 \mathrm{~g} / \mathrm{kgMS}$ & $0,03 \mathrm{~g} / \mathrm{kgMS}$ \\
Cobalto & $0,26 \mathrm{~g} / \mathrm{kgMS}$ & $0,08 \mathrm{~g} / \mathrm{kgMS}$ \\
FDNi & $40,09 \%$ & $28,51 \%$ \\
FDAi & $34,78 \%$ & $21,14 \%$ \\
\hline
\end{tabular}

$\mathrm{FDNi}=$ fibra em detergente neutro indigestível; $\mathrm{FDA}=$ fibra em detergente ácido indigestível.

(2004) relataram digestibilidade ruminal da matéria seca de 73,2\% em animais alimentados com feno e mistura concentrada na proporção de 60:40\%, respectivamente; assim como Tibo et al. (2000) relataram a digestibilidade ruminal da matéria seca em $73,2 \%$ em dietas contendo $37,5 \%$ de concentrado. Os indicadores mais eficientes na estimativa do fluxo de matéria seca duodenal foram $\mathrm{FDNi}$ e $\mathrm{Co}$, com coeficientes de digestibilidade ruminal médio de $63,6 \%$ e $71,4 \%$, e fluxo duodenal diário estimado em 1,53 e $1,18 \mathrm{~kg}$ de matéria seca, respectivamente.

$\mathrm{OCr}_{2} \mathrm{O}_{3}$ apesar de ser o indicador externo mais utilizado nas pesquisas de nutrição animal também é o indicador que mais apresenta resultados contraditórios entre as pesquisas, possivelmente, devido a somatória de fatores como falta de distribuição homogênea entre as fases componentes da digesta, metodologias utilizadas para sua análise, perfil excretório nas fezes, entre outros. Enquanto alguns pesquisadores conseguem sua recuperação completa outros não conseguem nem realizar sua determinação nas amostras. Além desse fato, há também o perfil excretório deste indicador, de acordo com Kozloski et al. (2006) não existe uma estabilidade em sua excreção ao longo do dia, o que poderia inter- 
ESTIMATIVA DO FLUXO DE DIGESTA EM BOVINOS DE CORTE

Tabela II. Médias de fluxo duodenal de MS (kg/dia) e digestibilidade ruminal da matéria seca estimadas pelo método de único e duplo indicador. (Dry matter duodenal flow (kg/day) and ruminal apparent digestibility means estimated by single and double marker methods).

\begin{tabular}{lcccc}
\hline Indicador $^{1}$ & $\begin{array}{c}\text { Fluxo duodenal } \\
\text { (kgMS/dia) }\end{array}$ & CV\% & $\begin{array}{c}\text { Digestibilidade } \\
\text { ruminal(\%) }\end{array}$ & CV\% \\
\hline Método de único indicador & & & & \\
$\mathrm{Cr}_{2} \mathrm{O}_{3}$ & $2,8^{\mathrm{ABC}}$ & 20,7 & $32,3^{\mathrm{CDE}}$ & 34,6 \\
$\mathrm{FDNi}$ & $1,6^{\mathrm{D}}$ & 19,4 & $63,6^{\mathrm{B}}$ & 7,7 \\
$\mathrm{FDAi}$ & $0,8^{\mathrm{E}}$ & 18,7 & $80,2^{\mathrm{A}}$ & 3,1 \\
$\mathrm{Co}$ & $1,2^{\mathrm{DE}}$ & 14,8 & $71,4^{\mathrm{AB}}$ & 6,6 \\
$\mathrm{Yb}$ & $2,5^{\mathrm{C}}$ & 4,8 & $38,6^{\mathrm{C}}$ & 30,5 \\
$\mathrm{C}_{31}$ & $3,4^{\mathrm{AB}}$ & 21,9 & $19,3^{\mathrm{DE}}$ & 97,8 \\
$\mathrm{C}_{32}$ & $2,5^{\mathrm{C}}$ & 15,0 & $39,8^{\mathrm{C}}$ & 32,2 \\
$\mathrm{C}_{33}$ & $3,4^{\mathrm{A}}$ & 20,6 & $17,5^{\mathrm{E}}$ & 109,3 \\
$\mathrm{C}_{35}$ & $3,4^{\mathrm{A}}$ & 20,0 & $17,3^{\mathrm{E}}$ & 102,7 \\
$\mathrm{C}_{36}$ & $2,8^{\mathrm{ABC}}$ & 15,9 & $30,9^{\mathrm{CDE}}$ & 52,8 \\
Método de duplo indicador & & & & \\
Co+Cr & $2,8^{\mathrm{ABC}}$ & 19,8 & $34,1^{\mathrm{CDE}}$ & 25,8 \\
Co+FDNi & $1,4^{\mathrm{DE}}$ & 6,1 & $65,9^{\mathrm{AB}}$ & 11,3 \\
CV\% & 19,3 & & 30,4 & \\
\hline
\end{tabular}

Médias seguidas de letras maiúsculas iguais nas colunas não diferem entre si, pelo Teste de Tukey ( $p>0,05) ; C V \%=$ coeficiente de variação.

$\mathrm{FDNi}=$ fibra em detergente neutro indigestível; $F D A i=$ fibra em detergente ácido indigestível; $E D T A ; \mathrm{Yb}=$ acetato de itérbio; $\mathrm{C}_{31}, \mathrm{C}_{32}, \mathrm{C}_{33}, \mathrm{C}_{35}, \mathrm{C}_{36}=$ alcanos internos e externos; $\mathrm{Co}+\mathrm{Cr}=$ cobaltoEDTA associado ao óxido de crômio; $\mathrm{Co}+\mathrm{FDNi}=$ cobaltoEDTA associado a fibra em detergente neutro indigestível.

ferir também na estimativa do fluxo de digesta duodenal, apesar das coletas de digesta serem divididas igualmente entre os períodos vespertino e noturno. Berchielli et al. (1998) relataram baixa recuperação para o $\mathrm{Cr}_{2} \mathrm{O}_{3}$ que superestimou os valores obtidos para o fluxo de MS duodenal concordando com os resultados obtidos por Freitas et al. (2002).

Quando se utiliza a técnica de único indicador na estimativa do fluxo de digesta e seus constituintes, é assumido que a composição da digesta coletada na cânula é idêntica à da digesta que está passando pela cânula. Contudo, esse requisito não pode ser garantido em amostras coletadas em cânulas simples, como as do tipo T. Segundo Faichney (1980), amostras de digesta que deixavam o abomaso de ovelhas recebendo dieta peletizada de leucena e aveia possuía em sua composição $0,055 \mathrm{~g}$ de matéria seca, enquanto a digesta presente no abomaso continha $0,151 \mathrm{~g}$ de matéria seca, isto é, não possuíam composição semelhante. Isso porque existe a tendência da digesta em se separar, e, na coleta com esse tipo de cânula dificilmente são obtidas amostras que contenham a parte fluida e particulada nas mesmas proporções que na digesta passante pela cânula. Para corrigir este problema, Faichney (1975) propôs o uso de dois indicadores que não necessitavam estar associado exclusivamente a uma das fases, o método de duplo indicador. Nesse método assume-se que os indicadores estejam distribuídos uniformemente dentro das fases (líquida e particulada) e a partir do cálculo de um fator de reconstituição, calculado, há a possibilidade de estimar o fluxo verdadeiro de digesta 
mesmo em amostras efetuadas por coleta em cânulas do tipo T.

No presente trabalho, a associação do indicador $\mathrm{Co}+\mathrm{Cr}$ (método de duplo indicador) superestimou o fluxo duodenal $(2,8 \mathrm{~kg}$ $\mathrm{MS} /$ dia) com coeficiente de digestibilidade de $34,1 \%$ enquanto a estimativa biologicamente mais adequada para a estimativa do fluxo duodenal foi obtida com a combinação $\mathrm{Co}+\mathrm{FDNi}$, com coeficiente de digestibilidade ruminal médio de $65,9 \%$ e fluxo diário estimado em $1,4 \mathrm{~kg} \mathrm{MS} / \mathrm{dia}$, dessa forma, esta combinação apresentou os melhores resultados.

Segundo Titgemeyer (1997), valores de digestão ruminal e no trato total dependem da dieta consumida e do nível de ingestão e, em dietas basicamente constituídas de forragem, a digestão ruminal é quase igual à digestão no trato total. Como o parâmetro fluxo duodenal não é exato e nem medido em sua totalidade pela cânula do tipo $\mathrm{T}$, as amostragens da digesta permitem apenas estimativas, não sendo descartados valores que apresentem digestibilidade ruminal biologicamente adequada em função da dieta oferecida. Desta forma, os fluxos estimados com FDNi, Co e Co+FDNi observados, com médias de 63,6; 71,4 e 65,9\% de digestibilidade ruminal, respectivamente, considerados, portanto, biologicamente adequados, permitem a afirmação de que estes indicadores são adequados na estimativa deste parâmetro em animais recebendo dieta exclusiva de forragem.

Os dados referentes à estimativa de fluxo de digesta omasal e digestibilidade ruminal estimada com os indicadores são apresentados na tabela II.

Ahvenjarvi et al. (2003) apresentam como benefícios da técnica de coletas de amostras pelo omaso a redução da intervenção cirúrgica nos animais e também o fato da coleta neste local do trato gastrintestinal ser menos afetada por secreção e digestão das células microbianas. Entretanto, cita também que uma desvantagem da técnica do omaso é que as amostras coletadas não são representativas da digesta que deixa o rúmen e passa para o omaso (Huhtanen et al., 1997; Ahvenjarvi et al., 2000), com maiores desvios em relação a digesta verdadeira quando comparada aos resultados obtidos nas coletas de duodeno.

$\mathrm{O}$ indicador externo $\mathrm{Cr}_{2} \mathrm{O}_{3}$ superestimou o fluxo de digesta omasal além de apresentar alto coeficiente de variação dos dados na estimativa da digestibilidade ruminal, fato que o caracteriza como um indicador inadequado na estimativa do fluxo omasal de matéria seca em bovinos recebendo dieta exclusiva de forragem. Resultado semelhante com o uso do $\mathrm{Cr}_{2} \mathrm{O}_{3}$ na estimativa de fluxo omasal foi observado por Dias et al. (2007), apresentando baixo coeficiente de digestibilidade. $\mathrm{O}$ fato da dificuldade de obtenção de amostra representativa de digesta nesse compartimento do TGI animal também pode ter comprometido a eficiência do indicador externo $\mathrm{Cr}_{2} \mathrm{O}_{3}$.

Já os indicadores internos FDAi e FDNi, no método de único indicador, apresentaram resultados biologicamente adequados na estimativa do fluxo omasal. Detmann et al. (2001) observaram que os indicadores internos possuem um perfil excretório mais estável em relação aos indicadores externos, como observado neste experimento.

A estimativa de fluxo de digesta omasal e digestibilidade ruminal estimada com os indicadores são apresentadas na tabela III.

Os resultados observados na tabela III permitem inferir que os indicadores FDNi, FDAi e a combinação Co+FDAi foram eficientes na estimativa do fluxo omasal de digesta. Dias et al. (2007) ao compararem indicadores na estimativa da digestibilidade parcial em bovinos concluíram que estes mesmos indicadores supracitados podem ser utilizados na estimativa de parâmetros através da coleta omasal de digesta. Ahvenjärvi et al. (2003) relataram que o método de único indicador não foi adequado na estimativa do fluxo de digesta omasal, sendo os métodos de duplo e triplo indicador os mais apropriados. No presente 
ESTIMATIVA DO FLUXO DE DIGESTA EM BOVINOS DE CORTE

Tabela III. Médias de fluxo omasal e digestibilidade ruminal da matéria seca estimadas pelo método de único e duplo indicador. (Dry matter omasal flow and ruminal apparent digestibility means estimated by single and double marker methods).

\begin{tabular}{lcccc}
\hline Indicador $^{1}$ & $\begin{array}{c}\text { Fluxo omasal } \\
\text { (kg MS/dia) }\end{array}$ & CV\% & $\begin{array}{c}\text { Digestibilidade ruminal } \\
\text { MS (\%) }\end{array}$ & CV\% \\
\hline Método de único indicador & & & $11,6^{\mathrm{C}}$ & 136,1 \\
$\mathrm{Cr}_{2} \mathrm{O}_{3}$ & $3,7^{\mathrm{A}}$ & 15,9 & $58,2^{\mathrm{B}}$ & 9,3 \\
$\mathrm{FDNi}$ & $1,8^{\mathrm{B}}$ & 19,5 & $73,4^{\mathrm{A}}$ & 4,6 \\
$\mathrm{FDAi}$ & $1,1^{\mathrm{C}}$ & 18,0 & & \\
Método de duplo indicador & & & 39,1 & 23,3 \\
Co+FDAi & $1,9^{\mathrm{B}}$ & & 22,2 & \\
CV\% & 23,9 & & \\
\hline
\end{tabular}

Médias seguidas de letras maiúsculas iguais nas colunas não diferem entre si, pelo Teste de Tukey $(p>0,05) ; C V \%=$ coeficiente de variação; FDNi= fibra em detergente neutro indigestível; FDAi=fibra em detergente ácido indigestível; $\mathrm{Co}+\mathrm{FDA}=$ cobaltoEDTA associado a fibra em detergente ácido indigestível.

trabalho os resultados observados demonstram que os indicadores internos, no método de único indicador, não diferiram estatisticamente da associação entre o Co e a FDAi (Co+FDAi), no método de duplo indicador.

Em decorrência da incapacidade para validar estimativas de fluxo digestivo com medidas de referência verdadeiras, os méritos e desvantagens dos métodos dos marcadores têm sido avaliados sobre a base de considerações teóricas (Ahvenjärvi et al., 2003). Contudo, a incerteza na comparação entre diferentes valores de fluxo de nutrientes em um mesmo segmento do trato gastrintestinal de ruminantes, estimados por indicadores e estatisticamente semelhantes como neste experimento, permite ainda algumas análises na busca por indicadores mais confiáveis. Entre essas análises, a partição da digestão da fibra entre desaparecimento ruminal e pós-ruminal pode ser usada como indicativo para compreender se o comportamento de um marcador é adequado (Berchielli et al., 2005). Quando o fluxo de digesta total para o duodeno é subestimado, a digestão ruminal da fibra poderá ser superestimada e erroneamente indica que grande porção da fibra desapa- rece no rúmen. Em forragens de baixa qualidade, quase toda a digestão da fibra ocorre no rúmen (Köster et al., 1996). Com forragens de alta qualidade e alguns subprodutos com alto teor de fibra, uma porção da fibra potencialmente digestível pode sair do rúmen e ser digerida pós-rúmem, particularmente se a degradação de fibra foi inibida por adição de gordura à dieta (Pantoja et al., 1994), no entanto, em ruminantes alimentados com forragens e subprodutos de boa qualidade, acima de $80 \%$ da digestão da fibra ocorrem no rúmen.

Na tabela IV é apresentada uma análise para avaliação de qual indicador ou combinação de indicadores permite estimar adequadamente a digestibilidade ruminal da fibra em detergente neutro.

Considerando-se a digestibilidade ruminal da FDN de $100 \%$, isto é, partindo-se do pressuposto que toda a digestibilidade da fração fibrosa ocorre no rúmen, é possível observar que apenas as combinações de indicadores, (o método de duplo indicador) estima de forma adequada o fluxo de digesta duodenal ou omasal, isto é, não difere de $100 \%$ a digestão dessa fração da fibra no rúmen quando o valor de fluxo é estimado com este método de coleta. Com base na 
Tabela IV. Digestibilidade ruminal da fibra em detergente neutro estimada através de diferentes indicadores obtidos por coleta de digesta duodenal ou omasal em bovinos alimentados com capim braquiária. (Ruminal digestibility of neutral detergent fiber estimated by different markers from duodenal or omasal sampling in steers fed palisade grass).

\begin{tabular}{lcr}
\hline Indicador $^{1}$ & \multicolumn{2}{c}{ Método de coleta } \\
& Duodenal & \multicolumn{1}{c}{ Omasal } \\
\hline $\mathrm{Cr}$ & $72,55 \pm 3,49^{*}$ & $50,55 \pm 4,45^{*}$ \\
$\mathrm{Yb}$ & $79,13 \pm 3,28^{*}$ & $-145,45 \pm 17,33^{*}$ \\
$\mathrm{FDNi}$ & $75,31 \pm 0,70^{*}$ & $70,65 \pm 1,11^{*}$ \\
$\mathrm{FDAi}$ & $54,17 \pm 2,83^{*}$ & $81,37 \pm 0,73^{*}$ \\
$\mathrm{Co}+\mathrm{Cr}$ & $74,54 \pm 3,59^{*}$ & $63,76 \pm 6,56^{*}$ \\
Co+Yb & $-96,72 \pm 32,0^{*}$ & $-194,45 \pm 42,54$ \\
Co+FDNi & $103,34 \pm 1,53^{\mathrm{NS}}$ & $58,87 \pm 4,37^{*}$ \\
Co+FDAi & $128,69 \pm 2,15^{*}$ & $97,54 \pm 5,70^{\mathrm{NS}}$ \\
\hline
\end{tabular}

'Digestibilidade ruminal da fibra em detergente neutro testada para a probabilidade da média ser igual a $100 \%$ pelo $t$-test a $5 \%$. Se não significativo (NS), média da digestibilidade ruminal da FDN não difere de $100 \%(p>0,05) ;{ }^{*} p<0,001$.

digestibilidade ruminal da fibra apenas a combinação Co+FDNi não diferiu estatisticamente de $100 \%$ no cálculo do fluxo duodenal enquanto a combinação Co+FDAi não foi diferente de $100 \%$ de digestibilidade ruminal da fração fibrosa para o fluxo duodenal de digesta.

Os resultados obtidos nesta análise (tabela IV), baseada na digestibilidade ruminal da FDN, corroboram os obtidos por Ahvenjärvi et al. (2003), onde o método de único indicador foi considerado ineficiente. Considerando-se, portanto, apenas o método de duplo indicador como eficiente na estimativa do fluxo de nutrientes, duodenal ou omasal, apesar de em alguns casos não haver diferença estatística $(\mathrm{p}<0,05)$ entre as digestibilidades ruminal da MS (tabela II e III) entre os métodos de único e duplo indicador.

Possivelmente, a coleta pelo método de duplo indicador, que considera a digesta passante pela cânula diferente da digesta verdadeira e utiliza fatores de correção para estimativa do fluxo da mesma, permite uma estimativa mais adequada, corrigindo, portanto, possíveis recuperações incompletas dos indicadores, mesmo que ambos os indicadores não tenham sido completamente recuperados no compartimento em questão.

Na tabela V são apresentadas as médias de fluxo duodenal e omasal de matéria seca em bovinos alimentados com capim marandu.

Comparando-se os valores obtidos para fluxo de nutrientes coletado pelo duodeno com o valores obtidos na estimativa do fluxo coletados pelo omaso é possível observar que a combinação Co+FDAi na coleta omasal apresentou maior média para estimativa de fluxo (1,9 $\mathrm{kg}$ de MS/dia), estatisticamente semelhante $(p>0,05)$ apenas com a estimativa de fluxo duodenal realizada com o indicador FDNi $(1,6 \mathrm{~kg}$ de

Tabela $\boldsymbol{V}$. Médias de fluxo duodenal e fluxo omasal bovinos alimentados com capim braquiária. (Duodenal and omasal flow means in steers fed palisade grass).

\begin{tabular}{lc}
\hline Local coleta/Indicador & Fluxo (kgMS/dia) \\
\hline \multicolumn{2}{c}{ Fluxo duodenal } \\
FDNi & $1,6^{\mathrm{AB}}$ \\
$\mathrm{Co} \quad 1,2^{\mathrm{BC}}$ \\
Co+FDNi & $1,4^{\mathrm{BC}}$ \\
\multicolumn{1}{c}{ Fluxo omasal } & \\
FDNi & $1,8^{\mathrm{A}}$ \\
FDAi & $1,1^{\mathrm{C}}$ \\
Co+FDAi & $1,9^{\mathrm{A}}$ \\
CV\% & 23,2 \\
\hline
\end{tabular}

Médias seguidas de letras maiúsculas iguais nas colunas não diferem entre si, pelo teste de Tukey $(p>0,05)$.

$\mathrm{FDNi}=$ fibra em detergente neutro indigestível; $F D A i=$ fibra em detergente ácido indigestível; $\mathrm{Co}+\mathrm{FDNi}$ e $\mathrm{Co}+\mathrm{FDAi}=$ cobalto EDTA associado a fibra indigestível.

CV\%= coeficiente de variação. 


\section{ESTIMATIVA DO FLUXO DE DIGESTA EM BOVINOS DE CORTE}

MS/dia). Por sua vez, o fluxo de nutrientes coletado no duodeno e estimado pelo indicador Co e a combinação Co+FDNi são semelhantes $(p>0,05)$ a estimativa de fluxo de nutrientes coletado no omaso e estimado pelo indicador FDAi. Tais resultados além de corroborarem com a afirmação de que as amostras coletadas no omaso podem não ser representativas da digesta que deixa o rúmen (Huhtanen et al., 1997; Ahvenjärvi et al., 2000) demonstram ainda que a utilização de valores de fluxo duodenal em comparação aos valores de fluxo omasal deve ser cautelosa apesar de valores de fluxo semelhantes.

\section{CONCLUSÕES}

Os indicadores FDNi, Co e Co+FDNi podem ser utilizados como indicadores para

\section{BILIOGRAFIA}

Ahvenjarvi, S., Vanhatalo, A., Huhtanen, P. e Varvikko, T. 2000. Determination of reticulorumen and whole-stomach digestion in lacting cows by omasal canal or duodenal sampling. Brit. J. Nutr., 83: 67-77.

Ahvenjarvi, S., Vanhatalo, A., Shingfield, K.J.e Huhtanen, P. 2003. Determination of digesta flow entering the omasal canal of dairy cows using different marker systems. Brit. J. Nutr., 90: 41-52.

AOAC. 1990. Official methods of analysis. $15^{\mathrm{a}} \mathrm{ed}$. Arlington. Kenneth Helrich. Association of Official Analytical Chemists. 1298 pp.

Berchielli, T.T., Rodriguez, N.M., Osorioneto, E. e Rocha, S.S. 1998. Comparação de indicadores de fase sólida para medir fluxo de matéria seca e matéria orgânica no duodeno. Arq. Bras. Vet. Zootecn., 50: 147-152.

Berchielli, T.T., Andrade, P. e Furlan, C.L. 2000. Avaliação de indicadores internos em ensaios de digestibilidade. Rev. Bras. Zootecn., 29: 830833.

Berchielli, T.T., Oliveira, S.G., Carrilho, E.N.V.M., Feitosa, J.V. e Lopes, A.D. 2005. Comparação de marcadores para estimativas de produção fecal e de fluxo de digesta em bovinos. Rev. Bras. Zootecn., 34: 987-996.

Deschamps, F.C. 1999. Implicações do período de estimativa do fluxo duodenal de matéria seca. Os indicadores FDNi, FDAi e Co+FDAi podem ser utilizados como indicadores para estimativa do fluxo omasal de matéria seca. Entretanto, ressalta-se que os indicadores internos são componentes naturais dos alimentos fibrosos, o que facilita seu fornecimento aos animais.

Entre os métodos, o de duplo indicador é recomendado para estimar os fluxos duodenal e omasal, respeitando-se a escolha correta do indicador para cada método.

\section{AGRADECIMENTOS}

Ao Prof. Dr. Alexandre Vaz Pires da ESALQ/USP, Prof. Dr. Juliano José de Resende Fernandes da UFG.

crescimento na composição química e digestão dos tecidos de cultivares de capim elefante. Rev. Bras. Zootecn., 28: 1178-1189.

Detmann, E., Paulino, M.F. Zervoudakis, J.T., Valadares Filho, S.C., Euclydes, R.F., Lana, R.P. e Queiroz, D.S. 2001. Cromo e indicadores internos na determinação do consumo de novilhos mestiços, suplementados, a pasto. Rev. Bras. Zootecn., 30: 1600-1609.

Vega, A.G. de e Poppi, D.P. 1997. Extent of digestion and rumen condition as factors affecting passage of liquid and digesta particles. J. Agri. Sci., 128: 207-215.

Dias, M., Detmann, E., Leao, M.I., Souza, S.M., Valadares Filho, S.C., Vasconcelos, A.M., Renno, L.N. e Valadares, R.F.D. 2007. Indicadores para estimativa da digestibilidade parcial em bovinos. Rev. Bras. Zootecn., 36: 689-697.

Faichney, G.J. 1975. The use of markers to partition digestion within the gastrointestinal tract. In: MacDonald, I.W. e Warner, A.A.I. (Eds.). Digestion and metabolism in the ruminant. University of New England Publishing Unit. Armidale. pp. 277-291.

Faichney, G.J. 1980. The use of markers to measure digesta flow from the stomach of sheep fed once daily. J. Agri. Sci., 94: 313-318. 
Faichney, G.J. 1993. Digesta flow. In: Forbes, J.M., France, J. (Eds). Quantitative aspects of ruminant digestion and metabolism. England. Commonwealth Agricultural Bureaux. Cambridge University Press. pp. 53-85.

Freitas, D., Berchielli, T.T., Silveira, R.N., Soares, J.P.G., Fernandes, J.J.R. e Pires, A.P. 2002. Produção fecal e fluxo duodenal de matéria seca e matéria orgânica estimados por meio de indicadores. Rev. Bras. Zootecn., 31: 15211530.

Huhtanen, P., Brotz, P.G. and Satter, L.D. 1997. Omasal sampling technique for assessing fermentative digestion in the forestomach of dairy cows. J. Animal Sci., 75: 1380-1392.

Koster, H.H., Cochran, R.C., Titgemeyer, E.C., Vanzan, E.S., Abdelgadir, I. and St-Jean, G. 1996. Effect of increasing degradation intake protein on intake and digestion of low-quality, tall-grass-prairie forage by beef cows. J. Animal Sci., 74: 2473-2481.

Kozloski, G.V., Perez Netto, D., Oliveira, L., Maixner, A.R., Leite, D.T., Maccari, M., Brondani, I.L., Samchez, L.M.B. e Quadros, F.L.F. 2006. Uso de óxido de cromo como indicador da excreção fecal de bovinos em pastejo: variação das estimativas em função do horário de amostragem. Ciênc. Rural, 36: 599-603.

Leao, M.I., Valadares Filho, S.C., Renno, L.N., Gonçalves, L.C., Cecon, P.R., Azevedo, J.A.G. e Valadares, R.F.D. 2004. Consumos e digestibilidades totais e parciais de matéria seca, matéria orgânica, proteína bruta e extrato etéreo em novilhos submetidos a três níveis de ingestão e duas metodologias de coleta de digestas abomasal e omasal. Rev. Bras. Zootecn., 33: 1604-1615.

Leao, M.I., Valadares Filho, S.C., Renno, L.N., Cecon, P.R., Azevedo, J.A.G., Gonçalves, L.C. e Valadares, R.F.D. 2005. Consumos e digestibilidades totais e parciais de carboidratos totais, fibra em detergente neutro e carboidratos não-fibrosos em novilhos submetidos a três níveis de ingestão e duas metodologias de coleta de digestas abomasal e omasal. Rev. Bras. Zootecn., 34: 670-678.

Mayes, R.W, Lamb, C.S and Golgrove, P.M. 1986. The use of dosed and herbage n-alkanes as markers for the determination of herbage intake.
J. Agri. Sci., 107: 161-170.

Olivan, M. and Osoro, K. 1999. Effect of temperature on alkane extraction from faeces and herbage. J. Agri. Sci., 132: 305-312.

Pantoja, J., Firkins, J.L., Eastridge, M.L. and Hull, B.L. 1994. Effects of fat saturation and source of fiber on site of nutrient digestion and milk production by lactating dairy cows. J. Dairy Sci., 77: 2314-2356.

Punia, B.S., Leibholz, J. and Faychney, G.J. 1988. Effects of level of intake and urea supplementation of alkali-treated straw on protozoal and bacterial nitrogen synthesis in the rumen and partition of digestion in cattle. Am. J. Agr. Res., 39: 1181-1194.

SAS. 1990. linstitute SAS/STAT User's guide. $5^{\text {th }}$ ed. Cary. 956 pp.

Silva, D.J. e Queiroz, A.C. 2002. Análise de alimentos: métodos químicos e biológicos. $3^{\underline{a}}$ ed. Editora UFV. Viçosa. $235 \mathrm{pp}$.

Tibo, G.C., Valadares Filho, S.C., Valadares, R.F.D. Coelho da Silva, J.F., Cecon, P.R., Leao, M.I. e Silva, R.B. 2000. Níveis de concentrado em dietas de novilhos mestiços F1 simentalxnelore 1. Consumo e digestibilidades. Rev. Bras. Zootecn., 29: 910-919.

Titgemeyer, E.C.1997. Design and interpretation of nutrient digestion studies. J. Animal Sci., 75: 2235-2247.

Úden, P., Colucci, P.E. and Van Soest, P.J. 1980. Investigation of chromium, cerium and cobalt as marker in digesta. Rate of passage studies. $J$. Sci. Food and Agri., 31: 635-632.

Valadare Filho, S.C., Silva, J.F.C., Leao, M.I., Valadares, R.F.D. e Castro, A.C.G. 1990. Digestão total e parcial da matéria seca, matéria orgânica e carboidratos em novilhos holandeses, nelores e búfalos mestiços. Rev. Bras. Zootecn., 19: 416-423.

Valiente, O.L., Delgado P., Vega, A. de e Guada, J.A. 2003. Validation of the $n$-alkane technique to estimate intake, digestibility, and diet composition in sheep consuming mixed grain:roughage diets. Aust. J. Agr. Res., 54: 693-702.

Van Soest, P.J., RobertsonT, J.B. and Lewis, B.A. 1991. Methods for dietary fiber, neutral detergent fiber, and nonstarch polyssacharides in relation to animal nutrition. J. Dairy Sci., 74: 3583-3597. 